\title{
FLT3 inhibitors: clinical potential in acute myeloid leukemia
}

REVIEW

This article was published in the following Dove Press journal:

OncoTargets and Therapy

3 February 2017

Number of times this article has been viewed

\author{
Marie-Anne Hospital ${ }^{1-3}$ \\ Alexa S Green ${ }^{1-3}$ \\ Thiago T Maciel ${ }^{4-7}$ \\ Ivan C Moura ${ }^{4-7}$ \\ Anskar Y Leung ${ }^{8}$ \\ Didier Bouscary ${ }^{1-3}$ \\ Jerome Tamburini ${ }^{1-3}$ \\ 'Département Développement, \\ Reproduction, Cancer, Institut \\ Cochin, Centre National de la \\ Recherche Scientifique (CNRS), Unité \\ Mixte de Recherche (UMR) 8I04, \\ Institut National de la Santé et de \\ la Recherche Médicale (INSERM) \\ UI016, ${ }^{2}$ Faculté de Médecine \\ Sorbonne Paris Cité, Université Paris \\ Descartes, ${ }^{3}$ Equipe Labellisée Ligue \\ Nationale Contre le Cancer (LNCC), \\ ${ }^{4}$ INSERM UMR I 163, Laboratory of \\ Cellular and Molecular Mechanisms \\ of Hematological Disorders and \\ Therapeutic Implications, ${ }^{5}$ Paris \\ Descartes - Sorbonne Paris Cité \\ University, ${ }^{6} \mathrm{CNRS}$ ERL 8254, Imagine \\ Institute, ${ }^{7}$ Laboratory of Excellence \\ GR-Ex, Paris, France; ${ }^{8}$ Department \\ of Medicine, Division of Hematology, \\ LKS Faculty of Medicine, The \\ University of Hong Kong, Hong Kong, \\ People's Republic of China
}

Correspondence: Jerome Tamburini Institut Cochin, Inserm UI0I6 and UMR8I04, 22 rue Mechain, 75014 Paris, France

Email jerome.tamburini@inserm.fr
Abstract: Acute myeloid leukemia (AML) is an aggressive hematopoietic malignancy that is cured in as few as $15 \%-40 \%$ of cases. Tremendous improvements in AML prognostication arose from a comprehensive analysis of leukemia cell genomes. Among normal karyotype AML cases, mutations in the FLT3 gene are the ones most commonly detected as having a deleterious prognostic impact. FLT3 is a transmembrane tyrosine kinase receptor, and alterations of the FLT3 gene such as internal tandem duplications (FLT3-ITD) deregulate FLT3 downstream signaling pathways in favor of increased cell proliferation and survival. FLT3 tyrosine kinase inhibitors (TKI) emerged as a new therapeutic option in FLT3-ITD AML, and clinical trials are ongoing with a variety of TKI either alone, combined with chemotherapy, or even as maintenance after allogenic stem cell transplantation. However, a wide range of molecular resistance mechanisms are activated upon TKI therapy, thus limiting their clinical impact. Massive research efforts are now ongoing to develop more efficient FLT3 TKI and/ or new therapies targeting these resistance mechanisms to improve the prognosis of FLT3ITD AML patients in the future.

Keywords: AML, FLT3, FLT3-ITD, tyrosine kinase, target therapy, resistance mechanisms

\section{Introduction}

Acute myeloid leukemia (AML) is an aggressive hematopoietic cancer arising from bone marrow immature myeloid progenitor cells transformed by recurrent genetic alterations that cooperate to induce cell proliferation and survival. These driver genetic events may include gene fusion (such as $P M L-R A R A$ ) or alterations of genes involved in epigenetic (eg, DNMT3A), metabolism (eg, IDH1/2), transcription (eg, NPMI), and signaling (eg, FLT3, fms-like tyrosine kinase 3) regulation. ${ }^{1,2}$ Among these genes, FLT3 is the one most frequently altered in AML: up to $30 \%$ of cases harbor an internal tandem duplication (FLT3-ITD), or a tyrosine kinase domain (TKD) amino acid substitution (FLT3-TKD) is seen in $5 \%-10 \%{ }^{3,4}$ Currently, only a minority of patients with AML are cured by highly intensive therapies that encompass high-dose cytotoxic chemotherapy and hematopoietic stem cell transplantation. ${ }^{5}$ The detection of a FLT3-ITD mutation is a hallmark of adverse prognosis in AML as less than $15 \%$ of these patients experience long-term survival despite intensive therapies. ${ }^{6}$ In contrast, the detection of a FLT3-TKD mutation does not have the same negative impact on survival. ${ }^{7}$ Hence, finding new therapeutic options for the FLT3-ITD AML patient represents a major goal for physicians and scientists since years. In this review, we will focus on a novel therapeutic modality, the FLT3 tyrosine kinase inhibitors (TKI), and discuss the mechanisms involved in the frequent clinical resistance to these molecules. 


\section{FLT3 mutations in AML FLT3 structure and function}

FLT3 belongs to a family of 58 protein tyrosine kinase receptors (TKR) characterized by an extracellular domain for ligand binding, a transmembrane helix, and a C-terminal intracytoplasmic part supporting the tyrosine kinase. ${ }^{8}$ FLT3 is a Class III TKR characterized by the presence of five immunoglobulin-like motifs within their extracellular part that are exclusively expressed in hematopoietic cells and induce the activation of intracellular signaling pathways such as PI3K/AKT or ERK/MAPK upon ligand binding (Figure 1). ${ }^{9}$ Mice knockout experiments revealed the critical role of FLT3 in normal hematopoiesis, as FLT3-/- hematopoietic progenitors fail to compensate for hematopoietic deficiency in lethally irradiated mice. ${ }^{10,11}$ Moreover, Boyer et a ${ }^{12}$ demonstrated that in mice, FLT3 is expressed on very immature hematopoietic progenitor cells lacking self-renewing potential but retaining capacity of differentiating into all hematopoietic lineages including megakaryocytes and erythrocytes, highlighting the critical role of FLT3 in the early stages of hematopoiesis.

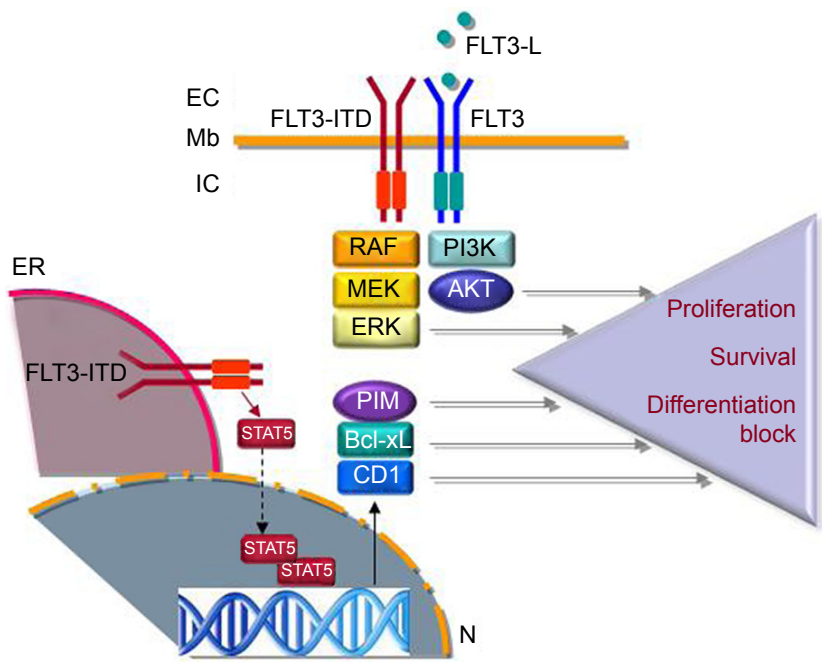

Figure I Schematic view of FLT3 and FLT3-ITD signaling.

Notes: FLT3 and FLT3-ITD receptors are expressed at the cell Mb. Their EC part is composed of five immunoglobulin-like domains that bind the FLT3-L. Their IC portion contains the TKD that supports the enzymatic activity of these receptors. FLT3 receptors are activated upon FLT3-L binding and, while remaining sensitive to FLT3-L, FLT3-ITD receptors are capable of activating downstream signaling pathways after FLT3-L-independent homodimerization. From the cell Mb receptors, several signaling pathways may be activated including the RAF/MEK/ERK and PI3K/AKT (the most studied). In contrast to FLT3 receptors, FLT3-ITD proteins may be found in the ER due to alteration of their glycosylation. Aberrant signaling from the ER activates STAT5 transcription factors that translocate into the nucleus $(N)$ and subsequently activate a transcriptional program leading to the accumulation of oncogenic proteins such as Pim kinases, Bcl-xL, or CDI. Signaling pathway activation from both $\mathrm{Mb}$ and ER contribute to cell proliferation and survival as well as to a differentiation block that contributes to AML propagation.

Abbreviations: Mb, membrane; EC, extracellular; FLT3-L, FLT3 ligand; IC, intracellular; TKD, tyrosine kinase domain; ER, endoplasmic reticulum; CDI, cyclin DI; AML, acute myeloid leukemia; Pim, proviral integration site.

\section{FLT3 in AML: clinical perspectives}

Early reports demonstrated an overexpression of FLT3 mRNA in AML and acute B-cell (but not T-cell) leukemia ${ }^{13}$ as well as an overexpression of FLT3 ligand (FLT3-L). ${ }^{14}$ However, a breakthrough in our understanding of AML pathophysiology came from the discovery of mutations within the FLT3 gene located on chromosome 13q12. Nakao et $\mathrm{a}^{15}$ identified FLT3 FLT3-ITD, detected in up to $30 \%$ of diagnosis AML cases, and then Yamamoto et $\mathrm{a}^{16}$ found point mutations within FLT3-TKD, leading to activating amino acid substitutions, which are detected in $5 \%-10 \%$ of AML samples.

From genome-wide sequencing studies, we learned that FLT3 is the most frequently mutated gene in AML, generally detected in samples with normal karyotype, and these mutations frequently co-occur with alterations of other genes such as DNMT3A or NPM1.,4 The presence of a FLT3-ITD mutation (but not of FLT3-TKD mutations) adversely impacts on prognosis, with a less than $20 \%$ long-term overall survival., In their recent, very large-scale genomic study on AML, Papaemmanuil et $\mathrm{al}^{3}$ observed a FLT3-ITD alteration in as much as $22 \%$ of a cohort of 1,540 AML patients. Interestingly, these mutations frequently co-occurred with NPM1 and DNMT3A mutations (39\%) and chromatin or RNA splicing gene mutations (15\%), and were also found to be associated with $\mathrm{t}(15 ; 17)$ and $\mathrm{t}(6 ; 9)$ translocations $(35 \%$ and $80 \%$, respectively). In this large study, FLT3-TKD mutations were found in less than $5 \%$ of the cohort, mostly co-occurring with MLL-PTD (1\%) and NPMI (3\%) abnormalities. This study unambiguously confirmed the adverse impact of FLT3-ITD mutations, particularly when co-occurring with both DNMT3A and NPM1 mutations, and observed an adverse impact of the gene-gene interaction between $M L L-P T D$ and FLT3-TKD mutations. ${ }^{3}$

While FLT3-ITD patients experience the same complete remission rate after induction therapy compared with other AML patients, they frequently relapse even after receiving allogeneic stem cell transplantation. The prognosis of relapsing patients is particularly poor as virtually none of the currently used therapies - including TKI that will be discussed later - induces long-term remissions. ${ }^{17} \mathrm{New}$ therapeutic approaches based on a better understanding of FLT3-ITD AML biology are, therefore, warranted to improve patient outcome.

\section{FLT3 in AML: biological perspectives}

Recent evidence suggests that AML arises from a clonal hematopoietic compartment referred to as preleukemic stem 
cells, which is expanded due to early genetic events such as DNMT3A mutations and thereafter transformed due to the occurrence of secondary driver mutations in other genes such as NPM1 or FLT3. ${ }^{18}$ From the comparison between AML genomes, we also learned that FLT3 mutations occur as secondary events during AML clonal evolution. ${ }^{2}$ While these data suggest that FLT3 mutations are secondary, late events during leukemogenesis, Chu et a ${ }^{19}$ demonstrated that FLT3-ITD drives a myeloproliferative disorder in mice due to proliferation and subsequent depletion of a quiescent long-term hematopoietic stem cell population, which was reverted by the FLT3 inhibitor sorafenib. A model to reconcile these findings may involve a preleukemic hematopoietic population amplified by initiating genetic lesions including DNMT3A, ASXL1, or TET2 mutations, which will be transformed into AML due to mutations - such as FLT3ITD - acquired later in the differentiation process. ${ }^{20}$ In the absence of a survival advantage during the early stages of hematopoiesis, the occurrence of a FLT3-ITD mutation may in contrast deplete the hematopoietic stem cell compartment, preventing the development of a FLT3-ITD-driven leukemic clone. ${ }^{19}$

FLT3-ITD mutation produces receptors with a constitutive tyrosine kinase activity due to homodimerization or heterodimerization with the normal FLT3 receptors. ${ }^{21}$ While FLT3 receptors induce signal transduction from the cell membrane, FLT3-ITD receptors are mostly located in the endoplasmic reticulum (ER) due to glycosylation defects leading to a distinct signaling pattern including the phosphorylation and activation of the STAT5 transcription factor. ${ }^{22}$ Interestingly, Chu et $\mathrm{al}^{19}$ have shown that FLT3 receptors are not detected at the cell surface of hematopoietic stem cell, but they found FLT3 mRNA and proteins and evidence for FLT3driven signal transduction in this cellular compartment, suggesting that intracellular ER-dependent FLT3-ITD signaling may be critical in disease propagation.

The constitutive activation of STAT5 by FLT3-ITD promotes AML cell proliferation and survival through the transactivation of target genes including BCL2L1, PIM1, $C D K N 1 A$, and $C C N D 1 .{ }^{23}$ Surprisingly, among STAT5 targets is the suppressor of cytokine signaling 1 gene encoding a negative regulator of cytokine-induced signaling, which promoted FLT3-ITD-induced myeloproliferative disease through escape from interferon growth inhibitory effects during cellular transformation. ${ }^{24}$ Recently, Chatterjee et $\mathrm{al}^{25}$ demonstrated that STAT5 nuclear translocation was due to a FAK/Rac1-Tiam1/PAK1 axis in FLT3-ITD and also in KIT D816V-positive cells and that targeting FAK, Tiam1, or PAK1 significantly reduced leukemia propagation in part through STAT5 inhibition.

FLT3-ITD also regulates myeloid cell differentiation, which is made evident by the observation of differentiation syndromes in AML patients treated by FLT3 inhibitors. ${ }^{26}$ In fact, FLT3-ITD blocks the activity of the $\mathrm{C} / \mathrm{EBP} \alpha$ transcription factor through direct phosphorylation of serine 21 and indirectly through CDK1 activation, inhibiting myeloid differentiation. ${ }^{27,28}$ In addition, other FLT3-ITD target genes are involved in differentiation including RGS2 and GADD45. ${ }^{29,30}$

Interestingly, metabolic pathways - ie, glycolysis and mitochondrial tricarboxylic acid cycle - recently emerged as new targets for cancer therapy. ${ }^{31}$ In FLT3-ITD AML cells, Alvarez-Calderon et $\mathrm{al}^{32}$ demonstrated that targeting mitochondrial metabolism using oligomycin A, an inhibitor of the mitochondrial ATP synthase, enhanced the antileukemic activity of the FLT3 TKI quizartinib against primary AML samples in vitro and in vivo in humanized patient-derived xenograft mouse models, suggesting that FLT3-ITD could induce an addiction of AML cells to mitochondrial metabolism.

Finally, recent data implicated a deregulation of oxidative metabolism in FLT3-ITD-induced leukemogenesis. FLT3-ITD receptors increase the expression of reactive oxygen species (ROS) due to increased nicotinamide adenine dinucleotide phosphate (NADPH) oxidase isoform 4 expression through STAT5 and also p22(phox), a small membranebound subunit of the NADPH oxidase complex. ${ }^{33,34}$ Besides DNA damage and genomic instability, ROS production may affect the function of signaling effectors as illustrated by Godfrey et al, ${ }^{35}$ showing that ROS overproduction due to FLT3-ITD inactivates the protein tyrosine phosphatase DEP-1 - a negative regulator of FLT3-ITD signaling through oxidation of its catalytic cysteine.

\section{FLT3 tyrosine kinase inhibitors}

The characterization of frequent FLT3 mutations in AML patients and their correlation to adverse clinical outcome in the case of FLT3-ITD prompted the search for FLT3 TKI. These TKI are heterocyclic compounds blocking FLT3 activity through competition with ATP at the TKD of FLT $3 .{ }^{36}$ While generally demonstrating in vitro activity against multiple tyrosine kinases, their efficacy in AML patients is markedly enhanced in FLT3-ITD cases and correlates with inhibition of FLT3 phosphorylation and downstream signaling relays. ${ }^{36}$ Comprehensive reviews on the use of FLT3 TKI in clinical trials have been recently published. ${ }^{17,36}$

Schematically, three categories of studies currently encompass FLT3 inhibitors in AML. First, studies using 
FLT3 TKI as monotherapy were reported mostly in a refractory/relapse context of FLT3-ITD AML. Some of them are already reported and show that, while of significant clinical activity, FLT3 TKI, such as quizartinib or sorafenib, fail to induce long-lasting remissions in this context, while some patients were successfully bridged to allogenic transplant after achieving TKI-induced remission. ${ }^{37,38}$ Second, several clinical trials were recently designed to combine a TKI to Vidaza, a hypomethylating agent with activity in AML, due to a possible synergistic activity of these compounds in FLT3-ITD AML. ${ }^{17}$ Third, important results recently came from clinical trials combining a TKI with conventional chemotherapy in AML. In a Phase II randomized trial (SORAML), 276 AML patients were treated by conventional chemotherapy plus sorafenib (400 mg twice daily, $n=134$ ) or plus placebo $(n=133)$, resulting in a $40 \%$ versus $22 \%$ 3 -years event-free survival in favor of the sorafenib group. In this study, a trend to improved survival was detected in patients treated by sorafenib when a FLT3-ITD was present, although the results were not statistically significant possibly due to the limited size of the cohort $(n=46) .{ }^{39}$ In a recent abstract, Stone et $\mathrm{al}^{40}$ reported the results of a Phase III study (RATIFY) that combined midostaurine or placebo to a standard chemotherapy in 717 FLT3-ITD AML patients. They showed that the addition of midostaurine extended the median overall survival from 25 to 75 months, which represents the first demonstration of a significant activity of a TKI in FLT3-ITD ${ }^{+}$AML in randomized trials. These results prompted the designation of midostaurine as a breakthrough therapy in FLT3-mutated AML by the US Food and Drug Administration in 2016. Finally, as posttransplant relapses have been successfully managed by the FLT3 TKI sorafenib, ${ }^{41,42}$ the idea emerged to administer a FLT3 TKI in patients achieving a complete remission after allogenic transplant. In fact, several recent studies report promising results using this maintenance approach, albeit follow-up intervals remain short. ${ }^{43-45}$ In a recent retrospective study, sorafenib posttransplant maintenance was associated with an improved 2-year progression-free survival and a reduced risk of relapse. ${ }^{46}$ However, these preliminary results warrant confirmation in prospective clinical trials, and several questions need to be addressed in this perspective such as the timing of TKI initiation and the duration of therapy. ${ }^{17}$

\section{Resistance to therapy in FLT3-ITD AML}

The presence of a FLT3-ITD mutation has a strong negative prognosis impact in AML due to an increased risk of relapse after chemotherapy. The current treatment guidelines in
FLT3-ITD AML have been recently reviewed by Konig and Levis ${ }^{17}$ and support the use of allogenic stem cell transplantation whenever possible in first remission to decrease the risk of relapse. For refractory/relapse patients, no guidelines are available and their survival is generally very short regardless of the therapeutic intervention used. ${ }^{17}$

\section{Resistance to cytotoxic chemotherapy}

Genomic pairwise comparisons between diagnosis and relapse AML samples revealed the expansion of a founding clone or subclone, which may acquire new oncogenic mutations at relapse. ${ }^{47}$ In FLT3-ITD AML, relapse-specific mutations were found at low frequency in several genes including FLT3 (FLT3-TKD mutations), WT1, ASXL1, MLL3, and FAT4, which may provide some insights on the mechanisms of resistance to chemotherapy. ${ }^{48}$ Interestingly, MLL3 and FAT4 were tumor suppressors in the FLT3-ITD context, as knockdown of their expression by RNA interference stimulated AML propagation including in mouse models, suggesting that inactivating mutations in these genes may have favored AML relapse after chemotherapy. ${ }^{48}$

Interestingly, FLT3-ITD AML samples collected at the time of relapse appear to be more sensitive to FLT3 TKI in vitro compared to diagnosis samples, suggesting that chemotherapy is selective for an AML clone addicted to FLT3-ITD signaling. ${ }^{49}$ This clonal selection might be directly favored by chemotherapy, which markedly enhance the production of FLT3-L by the bone marrow microenvironment and activate FLT3-ITD-dependent signaling pathways in AML cells. ${ }^{50}$

\section{Oncogene addiction to FLT3-ITD}

A significant number of reports have shown that FLT3 TKI frequently induce clinical responses in FLT3-ITD AML, including some complete responses, when used as a monotherapy. ${ }^{17}$ While some transient responses in blast count are reported in the absence of FLT3-ITD mutation, probability due to the activity of TKI against other kinases, complete responses are seen only in FLT3-ITD patients, attesting for an oncogenic addiction to FLT3-ITD in their AML cells. ${ }^{36}$ However, responses are generally lost within month, even when the TKI is given continuously. ${ }^{38}$ The addiction of AML cells to FLT3-ITD suggests that this deregulated TKR may represent a very attractive target for therapy, and in fact, important efforts were undertaken to understand the molecular basis of resistance to TKI in FLT3-ITD AML to develop new efficient therapeutic strategies in the future. The current state of the art of these resistance mechanisms is depicted in Figure 2. 


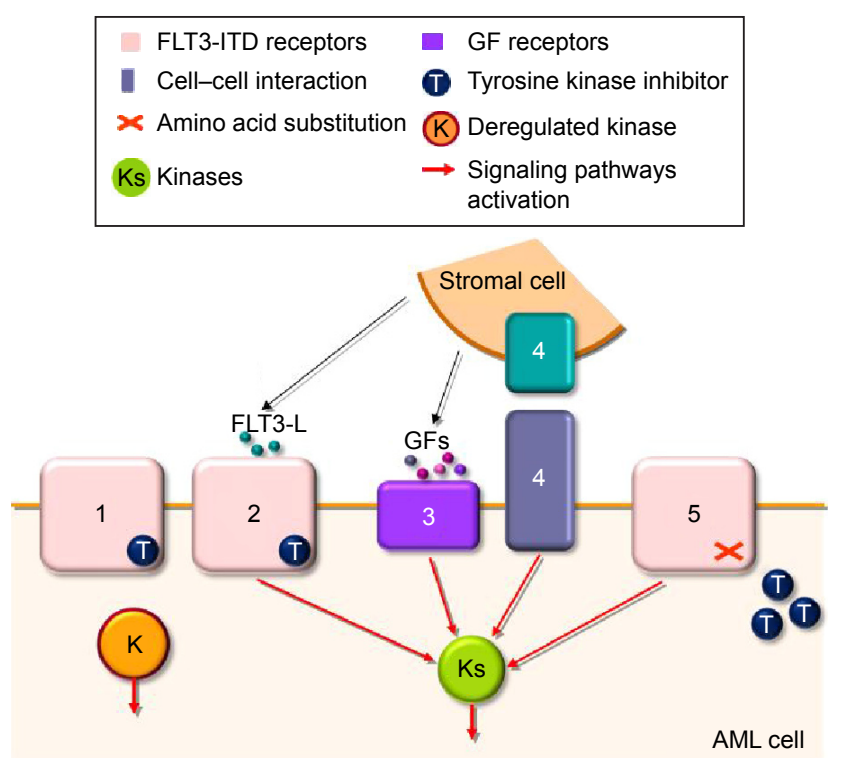

Figure 2 Overview of the resistance mechanisms to FLT3 TKI in AML. Notes: I) FLT3-ITD receptors are efficiently targeted by TKI (T) but bypass activation of oncogenic tyrosine kinases (SYK or $A X L)$ or serine/threonine kinases (Pim) reduce the activity of $\mathrm{TKI}$ and enhance $\mathrm{AML}$ cell proliferation and survival 2) FLT3-L overproduction from bone marrow stromal cells, for example, as observed in AML patients treated with cytotoxic chemotherapy, stimulates FLT3ITD receptors and reduces the efficacy of TKI. 3 and 4) Stromal cells from the bone marrow microenvironment may favor FLT3-ITD AML cell survival upon TKI therapy through the secretion of soluble cytoprotective factors that activate signaling pathways such as ERK (3) or through cell-to-cell interactions, involving, for example, integrins (4). 5) Amino acid substitutions within the FLT3-TKD (generally at position D835) decrease the affinity of TKI for their target receptors. The mechanisms hereby referenced from I to 5 result in the activation of intracellular signaling pathways such as ERK or STAT5 even in the presence of TKI, which explains why AML cells escape from their cytotoxic effects.

Abbreviations: TKI, tyrosine kinase inhibitors; AML, acute myeloid leukemia; FLT3-L, FLT3 ligand; Pim, proviral integration site.

\section{FLT3 allele burden}

FLT3-ITD allele burden is defined by the ratio between the mutated and the wild-type allele. A high $(>0.5)$ FLT3ITD burden is associated with an adverse prognosis after conventional therapy, even if allogenic stem cell transplant may benefit this category of AML patients. ${ }^{51,52}$ In contrast, the antileukemic activity of six different FLT3 TKI appeared superior in vitro against samples with a high FLT3-ITD allele burden. ${ }^{49}$ Interestingly in this report, FLT3 inhibition did not correlate to TKI-induced cytotoxicity in all cases, suggesting that some samples may not have been addicted to FLT3-ITDdriven signaling. ${ }^{49}$ The follow-up of FLT3 allele burden in the course of conventional or TKI therapy will be facilitated by new sensitive sequencing techniques, and the impact of minimal residual disease monitoring will probably be widely evaluated in FLT3-ITD AML in the future..$^{53,54}$

\section{Overexpression of oncogenic kinases}

The activation of bypass signaling pathways in response to target inhibition of an oncogenic signaling pathway is a well-recognized mechanism of resistance to therapy in cancer, as illustrated, for example, by the activation of CRAF in $B R A F$ V600-mutated cells treated with vemurafenib or by the activation of the MET tyrosine kinase in EGFR-mutated cells treated by gefitinib. ${ }^{55}$ In FLT3-ITD AML, the cytosolic tyrosine kinase SYK is overactivated and directly activates FLT3-ITD by direct binding and phosphorylation. Moreover, SYK appears critical for myeloid disease propagation in mice, with potential implications in resistance to TKI. ${ }^{56}$ AXL is a receptor tyrosine kinase overexpressed in many cancer types including in AML. Upon incubation with TKI, FLT3-ITD cells - and particularly cells that were resistant to TKI - demonstrated an increased expression and phosphorylation of AXL, and AXL inhibition by small molecules and by RNA interference facilitated the activity of FLT3 TKI in vitro. ${ }^{57}$

The impact expression of the PIM serine/threonine kinase family members have been extensively studied in cancer and AML. ${ }^{58,59}$ PIM kinases are FLT3-ITD targets dependent on - at least for PIM1 - STAT5 activity that controls several oncogenic relays including transcription, protein translation, cell cycle, apoptosis, and cell migration. ${ }^{58}$ Early reports showed that PIM kinases overexpression may contribute to FLT3-ITD-induced cell transformation and resistance to FLT3 TKI in vitro. ${ }^{60-62}$ Several groups reported a significant antileukemic activity of PIM inhibitors in preclinical models of AML, ${ }^{63-65}$ and currently a clinical trial is ongoing with the LGH447 pan-PIM kinase inhibitor in AML regardless of their FLT3 mutational status (NCT02078609). We recently observed that PIM kinases overexpression decreases the efficacy of FLT3 TKI due to a direct action of PIM kinases on FLT3-ITD receptors. In AML cells resistant to TKI, PIM kinase inhibition by RNA interference or by the pan-PIM kinase inhibitor LGB321 (similar to LGH447) restored the cytotoxic activity of FLT3 TKI against these cells, providing the molecular basis to understand the synergy between FLT3 and PIM kinase inhibition in FLT3-ITD ${ }^{+}$AML. ${ }^{66}$ In fact, the SGI-1776 compound is a dual PIM/FLT3 inhibitor that reached a clinical trial development stage. Unfortunately, unanticipated cardiac toxicity - possibly due to an off-target activity against the hERG potassium channel - resulted in withdrawal of SGI-1776 from clinical use ${ }^{67}$ Future development of dual PIM/FLT3 inhibitors might be of great interest after TKI failure in FLT3-ITD AML patients.

\section{FLT3-L}

While the expression of FLT3 receptors is restricted to hematopoietic cells, FLT3-L is expressed by a wide variety of cells, particularly by bone marrow stroma and $\mathrm{T}$ lymphocytes 
after chemotherapy. ${ }^{50}$ In fact, increased levels of FLT3-L significantly impaired the ability of FLT3 TKI to inhibit FLT3-ITD activity and induce cytotoxicity in FLT3-ITD AML ${ }^{50}$ As FLT3-L levels gradually increased after repeated cycles of chemotherapy, these results suggested to combine chemotherapy with a TKI frontline, an approach that seems fruitful in the clinics as attested by the recent results of the RATIFY clinical trial as discussed earlier. ${ }^{40}$ In addition, evidence suggests that, in contrast to cytotoxic chemotherapy, treatment with hypomethylating agents such as 5-azacytidine may prevent the release of FLT3-L. On the basis of these observations, several clinical trials are currently ongoing combining Vidaza to different TKI molecules in FLT3-ITD AML. ${ }^{17}$ An interesting approach to bypass FLT3-L-induced resistance to therapy is the development of anti-FLT3 antibodies. These antibodies bind FLT3 regardless the concentration of FLT3-L and induce cytotoxicity mainly through antibody-dependent cell-mediated cytotoxicity. ${ }^{68}$ FLYSYN is such a therapeutic antibody that will be used in clinical trials to target minimal residual disease in AML in complete remission (NCT02789254).

\section{Microenvironment}

The bone marrow microenvironment has long been recognized as a feeder for hematopoietic cancer cell growth and has been involved in resistance to FLT3 TKI in FLT3-ITD AML. ${ }^{69}$ Soluble molecule secretion as well as cellular interactions between AML and stromal cells was reported as cytoprotective factors toward AML cells including against TKI, particularly through a sustained activity of signaling pathways such as ERK. ${ }^{70}$ A recent report also suggests an important role for integrin $\alpha v \beta 3$ in activating $\beta$-catenin signaling in FLT3-ITD AML cells, promoting resistance to FLT3 TKI. ${ }^{71}$ Clinical use of TKI revealed a differential activity of these molecules between the blood and the bone marrow compartment. First-generation FLT3 TKI frequently produced a peripheral blast cell clearance, while the bone marrow blast cells were generally persistent. ${ }^{17}$ Second-generation TKI more frequently induced complete bone marrow blast cell clearance, but occurring later compared to the blood compartment (several weeks compared to few days). In fact, bone marrow blast cell clearance seems to be due to progressive myeloid differentiation rather than apoptosis with the implication of $\mathrm{C} / \mathrm{EBP} \alpha$ downstream of FLT3-ITD inhibition. ${ }^{26}$ These data suggest that leukemia-permissive interactions with the bone marrow microenvironment are important mediators of FLT3 TKI resistance for which therapeutic strategies must be refined by combining TKI to agents that target these interactions, such as the CXCR4 inhibitor plerixafor.

\section{FLT3 mutation}

FLT3 TKIs are small molecules that bind to the TKD through establishing hydrogen bond interactions. The TKI/kinase complex is thereby stabilized, which prevents ATP from reaching its binding site. ${ }^{72}$ Mutations occurring within the binding site of TKI may reduce their affinity for these molecules, hence reducing their antileukemic potential. This therapeutic resistance mechanism by modification of the target is commonly found in bacteria with acquired resistance to antibiotics and has been widely studied in the face of resistance to TKI in chronic myelogenous leukemia (CML) ${ }^{55}$ While occurring in virtually all cases of TKI resistance in CML, with a particular mention of the T315I "gatekeeper" mutation preventing the binding of most TKI to ABL TKD, the detection of mutations within FLT3-TKD only recently emerged as an important mechanism of resistance to FLT3 TKI. ${ }^{36}$

These FLT3-TKD mutations may be detected at diagnosis in the absence of FLT3-ITD mutation in 5\%-10\% of AML cases, and the nature as well as the clinical impact of these mutations was recently reviewed by Leung et al. ${ }^{36}$ The same mutations may occur on FLT3-ITD alleles and induce amino acid substitutions within the kinase domain generally at position D835. ${ }^{38}$ The F691L amino acid substitution, which is found less frequently than D835 mutations, is a "gatekeeper" mutation similar to the T315I mutation found in CML and also induces a global resistance to FLT3 TKI. ${ }^{72}$ Other TKD mutations were reported at low frequency in FLT3-ITD relapse samples, and overall, Alvarado et $\mathrm{al}^{73}$ reported the occurrence of TKD mutations in $22 \%$ of relapse samples of FLT3-ITD AML patients treated by quizartinib, sorafenib, lestaurtinib, or KW-2449 in clinical trials. Smith et $\mathrm{al}^{74}$ reported FLT3-TKD mutations in all samples from patients relapsing from quizartinib therapy and demonstrated that these mutations were not detectable in diagnosis samples, suggesting a strong selective pressure against FLT3 during the course of TKI therapy that supported the notion of oncogene addiction to FLT3-ITD of AML cells, pinpointing FLT3-ITD as an important therapeutic target in AML.

However, the mechanisms by which these additional mutations confer resistance to TKI are not fully understood. TKD mutations may stabilize the FLT3 kinase domain in an open ATP-binding configuration that enhance FLT3 activity and reduce TKI binding. ${ }^{36}$ In fact, Type 2 TKI such as quizartinib or sorafenib bind the TKD in an inactive conformation state, while Type 1 TKI (midostaurine, crenolanib) 
may target both active and inactive conformations of tyrosine kinases. ${ }^{75}$ Interestingly, Smith et $\mathrm{l}^{74}$ reported that crenolanib, a Type 1 TKI with a strong selectivity for FLT3, was powerful to induce cytotoxicity in FLT3-ITD cells harboring TKD resistance mutations.

How to target these mutations arising after TKI therapy is a central question that should be addressed to improve long-term disease control. ${ }^{36}$ First, new, more potent TKI may be discovered, as discussed earlier in the case of crenolanib. In fact, several other TKI, such as AUZ454 or ponatinib, are currently being developed to challenge these FLT3-TKD mutations, with ponatinib being routinely used in $B C R-A B L$ T315I-mutated leukemia. ${ }^{76}$ Second, multikinase inhibitor or dual therapy with a FLT3 TKI and another inhibitor may be considered to circumvent signaling pathways activated in TKD-mutated AML cells. Following this concept, several approaches have been developed including combination of FLT3 inhibitors with mTOR, NFkB, MEK, or PI3K inhibitors and also new compounds with dual activity against FLT3 and PIM, JAK, or aurora kinases. ${ }^{36}$ Virtually any pathway involved in TKI resistance may be considered as a potential target for dual targeting, and a rigorous preclinical evaluation of these new therapeutic options is required to select the best candidates for further clinical development in the future.

\section{Other mechanisms}

Other resistance mechanisms may involve changes in the regulation of target genes and/or in activation of signaling pathways downstream of FLT3-ITD. ${ }^{38}$ Interestingly, microarray analysis on primary samples from FLT3-ITD AML patients who became refractory to sorafenib showed an upregulation of the TESC gene, which encodes a calciuminteracting protein with impact on intracellular $\mathrm{pH}$ regulation and resistance to sorafenib. ${ }^{77}$ Agnostic approaches such as those developed in this study may represent powerful approaches to identify new strategies to target TKI resistance mechanisms in FLT3-ITD AML cells. Interestingly, Lam et $\mathrm{al}^{78}$ recently identified (by using a high-content chemical screen) homoharringtonine, a plant alkaloid with cytotoxic activity in AML achieved through protein translation inhibition, as having a synergistic activity with sorafenib in vitro, in mouse models, and in a clinical trial conducted in patients with refractory/relapsed FLT3-ITD AML, some of whom were resistant to FLT3 TKI.

\section{Perspectives}

AML is currently cured in only a minority of patients, and this is even more so when an FLT3-ITD mutation is detected in leukemic blasts. Tremendous efforts led to the development of highly active and clinically pertinent FLT3 TKI, as accumulated evidences pinpointed that AML cells may be addicted to FLT3-ITD-dependent signaling. However, relapses are commonly observed after TKI therapy due to different resistance mechanisms that encompass FLT3 TKD amino acid substitutions, bypass activation of oncogenic kinases, and leukemia-permissive interactions with the bone marrow microenvironment. In contrast to CML in which resistance mechanisms appear to be uniform, the pattern of resistance mechanisms in FLT3-ITD AML is far from being fully understood and represent an active field of research. However, recent results from a Phase III clinical trial in which FLT3-ITD AML patients were treated with chemotherapy and midostaurine or placebo strongly suggested a survival benefit to FLT3 inhibition. Future strategies will include more potent and/or specific FLT3 inhibitors and integration into a global strategy in which TKI may be given in combination with chemotherapy and/or as a consolidation strategy, particularly after allogenic stem cell transplantation or even as a maintenance therapy, possibly combined to hypomethylating agents or other molecules for which a synergy may be found with FLT3 TKI.

\section{Disclosure}

The authors report no conflicts of interest in this work.

\section{References}

1. Murati A, Brecqueville M, Devillier R, Mozziconacci MJ, Gelsi-Boyer V, Birnbaum D. Myeloid malignancies: mutations, models and management. BMC Cancer. 2012;12:304.

2. Welch JS, Ley TJ, Link DC, et al. The origin and evolution of mutations in acute myeloid leukemia. Cell. 2012;150(2):264-278.

3. Papaemmanuil E, Gerstung M, Bullinger L, et al. Genomic classification and prognosis in acute myeloid leukemia. $N$ Engl J Med. 2016;374(23): 2209-2221.

4. Patel JP, Gönen M, Figueroa ME, et al. Prognostic relevance of integrated genetic profiling in acute myeloid leukemia. $N$ Engl J Med. 2012;366(12) 1079-1089.

5. Buchner T, Schlenk RF, Schaich M, et al. Acute Myeloid Leukemia (AML): different treatment strategies versus a common standard arm - combined prospective analysis by the German AML Intergroup. $J$ Clin Oncol. 2012;30(29):3604-3610.

6. Thiede C, Steudel C, Mohr B, et al. Analysis of FLT3-activating mutations in 979 patients with acute myelogenous leukemia: association with $\mathrm{FAB}$ subtypes and identification of subgroups with poor prognosis. Blood. 2002;99(12):4326-4335.

7. Janke H, Pastore F, Schumacher D, et al. Activating FLT3 mutants show distinct gain-of-function phenotypes in vitro and a characteristic signaling pathway profile associated with prognosis in acute myeloid leukemia. PLoS One. 2014;9(3):e89560.

8. Blume-Jensen P, Hunter T. Oncogenic kinase signalling. Nature. 2001; 411(6835):355-365.

9. Levis M. FLT3/ITD AML and the law of unintended consequences. Blood. 2011;117(26):6987-6990. 
10. Mackarehtschian K, Hardin JD, Moore KA, Boast S, Goff SP, Lemischka IR. Targeted disruption of the flk $2 / \mathrm{flt} 3$ gene leads to deficiencies in primitive hematopoietic progenitors. Immunity. 1995;3(1): 147-161.

11. Matthews W, Jordan CT, Wiegand GW, Pardoll D, Lemischka IR. A receptor tyrosine kinase specific to hematopoietic stem and progenitor cell-enriched populations. Cell. 1991;65(7):1143-1152.

12. Boyer SW, Schroeder AV, Smith-Berdan S, Forsberg EC. All hematopoietic cells develop from hematopoietic stem cells through Flk2/Flt3-positive progenitor cells. Cell Stem Cell. 2011;9(1):64-73.

13. Birg F, Courcoul M, Rosnet O, et al. Expression of the FMS/KIT-like gene FLT3 in human acute leukemias of the myeloid and lymphoid lineages. Blood. 1992;80(10):2584-2593.

14. Zheng R, Levis M, Piloto O, et al. FLT3 ligand causes autocrine signaling in acute myeloid leukemia cells. Blood. 2004;103(1):267-274.

15. Nakao M, Yokota $S$, Iwai $T$, et al. Internal tandem duplication of the flt3 gene found in acute myeloid leukemia. Leukemia. 1996;10(12): 1911-1918.

16. Yamamoto $\mathrm{Y}$, Kiyoi H, Nakano Y, et al. Activating mutation of D835 within the activation loop of FLT3 in human hematologic malignancies. Blood. 2001;97(8):2434-2439.

17. Konig H, Levis M. Targeting FLT3 to treat leukemia. Expert Opin Ther Targets. 2015;19(1):37-54.

18. Shlush LI, Zandi S1, Mitchell A, et al. Identification of pre-leukaemic haematopoietic stem cells in acute leukaemia. Nature. 2014;506(7488): 328-333.

19. Chu SH, Heiser D, Li L, et al. FLT3-ITD knockin impairs hematopoietic stem cell quiescence/homeostasis, leading to myeloproliferative neoplasm. Cell Stem Cell. 2012;11(3):346-358.

20. Hirsch P, Zhang Y, Tang R, et al. Genetic hierarchy and temporal variegation in the clonal history of acute myeloid leukaemia. Nat Commun. 2016;7:12475.

21. Kiyoi H, Towatari M, Yokota S, et al. Internal tandem duplication of the FLT3 gene is a novel modality of elongation mutation which causes constitutive activation of the product. Leukemia. 1998;12(9):1333-1337.

22. Schmidt-Arras D, Böhmer SA, Koch S, et al. Anchoring of FLT3 in the endoplasmic reticulum alters signaling quality. Blood. 2009;113(15): 3568-3576.

23. Choudhary C, Brandts C, Schwable J, et al. Activation mechanisms of STAT5 by oncogenic Flt3-ITD. Blood. 2007;110(1):370-374.

24. Reddy PN, Sargin B, Choudhary C, et al. SOCS1 cooperates with FLT3-ITD in the development of myeloproliferative disease by promoting the escape from external cytokine control. Blood. 2012;120(8): 1691-1702.

25. Chatterjee A, Ghosh J, Ramdas B, et al. Regulation of Stat 5 by FAK and PAK1 in oncogenic FLT3- and KIT-driven leukemogenesis. Cell Rep. 2014;9(4):1333-1348.

26. Sexauer A, Perl A, Yang X, et al. Terminal myeloid differentiation in vivo is induced by FLT3 inhibition in FLT3/ITD AML. Blood. 2012; 120(20):4205-4214.

27. Radomska HS, Alberich-Jordà M, Will B, Gonzalez D, Delwel R, Tenen DG. Targeting CDK1 promotes FLT3-activated acute myeloid leukemia differentiation through C/EBPalpha. J Clin Invest. 2012; 122(8):2955-2966.

28. Zheng R, Friedman AD, Levis M, Li L, Weir EG, Small D. Internal tandem duplication mutation of FLT3 blocks myeloid differentiation through suppression of C/EBPalpha expression. Blood. 2004;103(5): 1883-1890.

29. Perugini M, Kok CH, Brown AL, et al. Repression of Gadd45alpha by activated FLT3 and GM-CSF receptor mutants contributes to growth, survival and blocked differentiation. Leukemia. 2009;23(4):729-738.

30. Schwable J, Choudhary C, Thiede C, et al. RGS2 is an important target gene of Flt3-ITD mutations in AML and functions in myeloid differentiation and leukemic transformation. Blood. 2005;105(5): 2107-2114

31. Pusapati RV, Daemen A, Wilson C, et al. mTORC1-dependent metabolic reprogramming underlies escape from glycolysis addiction in cancer cells. Cancer Cell. 2016;29(4):548-562.
32. Alvarez-Calderon F, Gregory MA, Pham-Danis C, et al. Tyrosine kinase inhibition in leukemia induces an altered metabolic state sensitive to mitochondrial perturbations. Clin Cancer Res. 2015;21(6):1360-1372.

33. Stanicka J, Russell EG, Woolley JF, Cotter TG. NADPH oxidasegenerated hydrogen peroxide induces DNA damage in mutant FLT3expressing leukemia cells. J Biol Chem. 2015;290(15):9348-9361.

34. Jayavelu AK, Müller JP, Bauer R, et al. NOX4-driven ROS formation mediates PTP inactivation and cell transformation in FLT3ITD-positive AML cells. Leukemia. 2016;30(2):473-483.

35. Godfrey R, Arora D, Bauer R, et al. Cell transformation by FLT3 ITD in acute myeloid leukemia involves oxidative inactivation of the tumor suppressor protein-tyrosine phosphatase DEP-1/PTPRJ. Blood. 2012; 119(19):4499-4511.

36. Leung AY, Man CH, Kwong YL. FLT3 inhibition: a moving and evolving target in acute myeloid leukaemia. Leukemia. 2013;27(2):260-268.

37. Cortes JE, Kantarjian H, Foran JM, et al. Phase I study of quizartinib administered daily to patients with relapsed or refractory acute myeloid leukemia irrespective of FMS-like tyrosine kinase 3-internal tandem duplication status. J Clin Oncol. 2013;31(29):3681-3687.

38. Man CH, Fung TK, Ho C, et al. Sorafenib treatment of FLT3-ITD ${ }^{+}$ acute myeloid leukemia: favorable initial outcome and mechanisms of subsequent nonresponsiveness associated with the emergence of a D835 mutation. Blood. 2012;119(22):5133-5143.

39. Rollig C, Serve H, Hüttmann A, et al. Addition of sorafenib versus placebo to standard therapy in patients aged 60 years or younger with newly diagnosed acute myeloid leukaemia (SORAML): a multicentre, phase 2, randomised controlled trial. Lancet Oncol. 2015;16(16): 1691-1699.

40. Stone RM, Mandrekar S, Sanford BL, et al. The multi-kinase inhibitor midostaurin (M) prolongs survival compared with placebo (P) in combination with daunorubicin (D)/cytarabine (c) induction (ind), high-dose c consolidation (consol), and as maintenance (maint) therapy in newly diagnosed acute myeloid leukemia (AML) patients (pts) age 18-60 with FLT3 mutations (muts): an international prospective randomized (rand) P-controlled double-blind trial (CALGB 10603/RATIFY [Alliance]). Paper presented at: 57th Annual ASH Meeting; 2015; Orlando, FL.

41. De Freitas T, Marktel S, Piemontese S, et al. High rate of hematological responses to sorafenib in FLT3-ITD acute myeloid leukemia relapsed after allogeneic hematopoietic stem cell transplantation. Eur J Haematol. 2016;96(6):629-636.

42. Metzelder S, Wang Y, Wollmer E, et al. Compassionate use of sorafenib in FLT3-ITD-positive acute myeloid leukemia: sustained regression before and after allogeneic stem cell transplantation. Blood. 2009; 113(26):6567-6571.

43. Tarlock K, Chang B, Cooper T, et al. Sorafenib treatment following hematopoietic stem cell transplant in pediatric FLT3/ITD acute myeloid leukemia. Pediatr Blood Cancer. 2015;62(6):1048-1054.

44. Antar A, Kharfan-Dabaja MA, Mahfouz R, Bazarbachi A. Sorafenib maintenance appears safe and improves clinical outcomes in FLT3-ITD acute myeloid leukemia after allogeneic hematopoietic cell transplantation. Clin Lymphoma Myeloma Leuk. 2015;15(5):298-302.

45. Chen YB, Li S, Lane AA, et al. Phase I trial of maintenance sorafenib after allogeneic hematopoietic stem cell transplantation for fms-like tyrosine kinase 3 internal tandem duplication acute myeloid leukemia. Biol Blood Marrow Transplant. 2014;20(12):2042-2048.

46. Brunner AM, Li S, Fathi AT, et al. Haematopoietic cell transplantation with and without sorafenib maintenance for patients with FLT3-ITD acute myeloid leukaemia in first complete remission. $\mathrm{Br} J$ Haematol. Epub July 19, 2016.

47. Ding L, Ley TJ, Larson DE, et al. Clonal evolution in relapsed acute myeloid leukaemia revealed by whole-genome sequencing. Nature. 2012;481(7382):506-510.

48. Garg M, Nagata Y, Kanojia D, et al. Profiling of somatic mutations in acute myeloid leukemia with FLT3-ITD at diagnosis and relapse. Blood. 2015;126(22):2491-2501.

49. Pratz KW, Sato T, Murphy KM, Stine A, Rajkhowa T, Levis M. FLT3mutant allelic burden and clinical status are predictive of response to FLT3 inhibitors in AML. Blood. 2010;115(7):1425-1432. 
50. Sato T, Yang X, Knapper S, etal. FLT3 ligand impedes the efficacy of FLT3 inhibitors in vitro and in vivo. Blood. 2011;117(12):3286-3293.

51. Pratcorona M, Brunet S, Nomdedéu J, et al. Favorable outcome of patients with acute myeloid leukemia harboring a low-allelic burden FLT3-ITD mutation and concomitant NPM1 mutation: relevance to post-remission therapy. Blood. 2013;121(14):2734-2738.

52. Schlenk RF, Kayser S, Bullinger L, et al. Differential impact of allelic ratio and insertion site in FLT3-ITD-positive AML with respect to allogeneic transplantation. Blood. 2014;124(23):3441-3449.

53. Bibault JE, Figeac M, Hélevaut N, et al. Next-generation sequencing of FLT3 internal tandem duplications for minimal residual disease monitoring in acute myeloid leukemia. Oncotarget. 2015;6(26): 22812-22821.

54. Thol F, Kölking B, Damm F, et al. Next-generation sequencing for minimal residual disease monitoring in acute myeloid leukemia patients with FLT3-ITD or NPM1 mutations. Genes Chromosomes Cancer. 2012; 51(7):689-695.

55. Pagliarini R, Shao W, Sellers WR. Oncogene addiction: pathways of therapeutic response, resistance, and road maps toward a cure. EMBO Rep. 2015;16(3):280-296.

56. Puissant A, Fenouille N, Alexe G, et al. SYK is a critical regulator of FLT3 in acute myeloid leukemia. Cancer Cell. 2014;25(2):226-242.

57. Park IK, Mundy-Bosse B, Whitman SP, et al. Receptor tyrosine kinase Axl is required for resistance of leukemic cells to FLT3targeted therapy in acute myeloid leukemia. Leukemia. 2015;29(12): 2382-2389.

58. Brault L, Gasser C, Bracher F, Huber K, Knapp S, Schwaller J. PIM serine/threonine kinases in the pathogenesis and therapy of hematologic malignancies and solid cancers. Haematologica. 2010;95(6): 1004-10015.

59. Tamburini J, Green AS, Bardet V, et al. Protein synthesis is resistant to rapamycin and constitutes a promising therapeutic target in acute myeloid leukemia. Blood. 2009;114(8):1618-1627.

60. Kim KT, Baird K, Ahn JY, et al. Pim-1 is up-regulated by constitutively activated FLT3 and plays a role in FLT3-mediated cell survival. Blood. 2005;105(4):1759-1767.

61. Adam M, Pogacic V, Bendit M, et al. Targeting PIM kinases impairs survival of hematopoietic cells transformed by kinase inhibitor-sensitive and kinase inhibitor-resistant forms of Fms-like tyrosine kinase 3 and BCR/ABL. Cancer Res. 2006;66(7):3828-3835.

62. Agrawal S, Koschmieder S, Bäumer N, et al. Pim2 complements Flt3 wild-type receptor in hematopoietic progenitor cell transformation. Leukemia. 2008;22(1):78-86.

63. Chen LS, Redkar S, Taverna P, Cortes JE, Gandhi V. Mechanisms of cytotoxicity to Pim kinase inhibitor, SGI-1776, in acute myeloid leukemia. Blood. 2011;118(3):693-702.
64. Keeton EK, McEachern K, Dilman KS, et al. AZD1208, a potent and selective pan-Pim kinase inhibitor, demonstrates efficacy in preclinical models of acute myeloid leukemia. Blood. 2014;123(6):905-913.

65. Garcia PD, Langowski JL, Wang Y, et al. Pan-PIM kinase inhibition provides a novel therapy for treating hematologic cancers. Clin Cancer Res. 2014;20(7):1834-1845.

66. Green AS, Maciel TT, Hospital MA, et al. Pim kinases modulate resistance to FLT3 tyrosine kinase inhibitors in FLT3-ITD acute myeloid leukemia. Sci Adv. 2015;1(8):e1500221

67. Foulks JM, Carpenter KJ, Luo B, et al. A small-molecule inhibitor of PIM kinases as a potential treatment for urothelial carcinomas. Neoplasia. 2014;16(5):403-412.

68. Hofmann M, Große-Hovest L, Nübling T, et al. Generation, selection and preclinical characterization of an Fc-optimized FLT3 antibody for the treatment of myeloid leukemia. Leukemia. 2012;26(6):1228-1237.

69. Parmar A, Marz S, Rushton S, et al. Stromal niche cells protect early leukemic FLT3-ITD ${ }^{+}$progenitor cells against first-generation FLT3 tyrosine kinase inhibitors. Cancer Res. 2011;71(13):4696-4706.

70. Weisberg E, Liu Q, Nelson E, et al. Using combination therapy to override stromal-mediated chemoresistance in mutant FLT3-positive AML: synergism between FLT3 inhibitors, dasatinib/multi-targeted inhibitors and JAK inhibitors. Leukemia. 2012;26(10):2233-2244.

71. Yi H, Zeng D, Shen Z, et al. Integrin alphavbeta3 enhances betacatenin signaling in acute myeloid leukemia harboring Fms-like tyrosine kinase-3 internal tandem duplication mutations: implications for microenvironment influence on sorafenib sensitivity. Oncotarget. 2016.

72. Treiber DK, Shah NP. Ins and outs of kinase DFG motifs. Chem Biol. 2013;20(6):745-746.

73. Alvarado Y, Kantarjian HM, Luthra R, et al. Treatment with FLT3 inhibitor in patients with FLT3-mutated acute myeloid leukemia is associated with development of secondary FLT3-tyrosine kinase domain mutations. Cancer. 2014;120(14):2142-2149.

74. Smith CC, Lasater EA, Lin KC, et al. Crenolanib is a selective type I panFLT3 inhibitor. Proc Natl Acad Sci U S A. 2014;111(14):5319-5324.

75. Fathi AT. Emergence of crenolanib for FLT3-mutant AML. Blood. 2013;122(22):3547-3548

76. Smith CC, Lasater EA, Zhu X, et al. Activity of ponatinib against clinically-relevant AC220-resistant kinase domain mutants of FLT3ITD. Blood. 2013;121(16):3165-3171.

77. Man CH, Lam SS, Sun MK, et al. A novel tescalcin-sodium/hydrogen exchange axis underlying sorafenib resistance in FLT3-ITD ${ }^{+}$AML. Blood. 2014;123(16):2530-2539.

78. Lam SSY, Ho ESK, He B-L, et al. In vitro drug screening identified homoharringtonine as an effective adjunct for treatment of FLT3-ITD acute myeloid leukemia. Sci Transl Med. 2016;8(359):359ra129.
OncoTargets and Therapy

\section{Publish your work in this journal}

OncoTargets and Therapy is an international, peer-reviewed, open access journal focusing on the pathological basis of all cancers, potential targets for therapy and treatment protocols employed to improve the management of cancer patients. The journal also focuses on the impact of management programs and new therapeutic agents and protocols on

\section{Dovepress}

patient perspectives such as quality of life, adherence and satisfaction. The manuscript management system is completely online and includes a very quick and fair peer-review system, which is all easy to use. Visit http://www.dovepress.com/testimonials.php to read real quotes from published authors. 